\title{
Unique solvability of the CCD scheme for convection-diffusion equations with variable convection coefficients
}

\author{
Qinghe Wang ${ }^{1}$, Kejia Pan ${ }^{1}$ and Hongling $\mathrm{Hu}^{2 *}$
}

\section{"Correspondence:}

hhling625@163.com

${ }^{2}$ School of Mathematics and

Statistics, Key Laboratory of High

Performance Computing and

Stochastic Information Processing,

Hunan Normal University,

Changsha, P.R. China

Full list of author information is

available at the end of the article

\begin{abstract}
The combined compact difference (CCD) scheme has better spectral resolution than many other existing compact or noncompact high-order schemes, and is widely used to solve many differential equations. However, due to its implicit nature, very little theoretical results on the CCD method are known. In this paper, we provide a rigorous theoretical proof for the unique solvability of the CCD scheme for solving the convection-diffusion equation with variable convection coefficients subject to periodic boundary conditions.
\end{abstract}

MSC: $65 \mathrm{M} 06 ; 65 \mathrm{M} 32$

Keywords: Combined compact difference scheme; Convection-diffusion equation; Unique solvability; Variable coefficient; Periodic boundary conditions

\section{Introduction}

In many real physical applications, performing high-order and efficient numerical methods for solving the partial differential equations is essential. In particular, it is important to simultaneously solve the unknown function and its derivatives with high-order accuracy. For example, Lele [1] has shown that when the schemes involve not only the value of the function but also those of its derivatives, spectral-like resolution can be achieved while keeping a small stencil. Many attempts have been made to develop such schemes involving both the unknown function and its derivatives. Among these methods, the three-point sixorder combined compact difference scheme $(C C D)$ proposed by Chu and Fan [2-4] is well known and popular for its high efficiency. The CCD scheme, which can be regarded as an extension of the standard Pade schemes as discussed by Lele [1], allows us to conveniently handle the differential equations with variable coefficients subject to Robin boundary conditions. When using the CCD method to solve the differential equations, the equation is assumed to be valid at the boundary, and the first and second derivatives together with the function values of unknowns at grid points are computed simultaneously [2]. Fourier analysis shows that the CCD scheme is more accurate than many other compact or noncompact schemes [2]. Since its appearance, there has been a lot of research discussing the application and improvement of the method [5-15].

The CCD method is originally proposed to solve second-order linear ordinary differential equations [2]. For multi-dimensional evolution problems, we can employ alternat-

(c) The Author(s) 2018. This article is distributed under the terms of the Creative Commons Attribution 4.0 International License (http://creativecommons.org/licenses/by/4.0/), which permits unrestricted use, distribution, and reproduction in any medium, provided you give appropriate credit to the original author(s) and the source, provide a link to the Creative Commons license, and indicate if changes were made. 
ing direction implicit (ADI) technique to convert it into a series of one-dimensional (1D) problems, which can be solved efficiently by the CCD scheme [16-22]. Lee et al. [23] developed a CCD method for directly solving the general two-dimensional (2D) linear partial differential equation with a mixed derivative. Fractional differential equations have gained considerable importance due to their varied applications in many fields of sciences and engineering. Recently, the numerical estimation of fractional differential equations has been discussed in the existing literature [24-31].

However, due to its implicit nature, very little theoretical results on the CCD method are known. Zhang [32] derived the truncation error representation of the CCD scheme when applied to 1D convection-diffusion equations and analyzed its oscillation property. Sengupta $[11,15]$ and $\mathrm{Yu}[12,13]$ carefully studied the dispersion-relation of the CCD scheme, and proposed some improved CCD methods. To our best knowledge, the solvability and convergence of the CCD scheme for solving convection-diffusion equations have not been obtained in the existing literature.

In this paper, we consider the following second-order linear ordinary differential equation:

$$
-\frac{\partial^{2} u}{\partial x^{2}}+a(x) \frac{\partial u}{\partial x}+b u=c(x), \quad 0<x<1,
$$

with periodic boundary condition, where $b$ is a positive constant, $a(x)$ and $c(x)$ are assumed to be sufficiently smooth. In this paper, we will provide a rigorous theoretical proof for the unique solvability of the CCD scheme for solving the above convection-diffusion equation subject to periodic boundary conditions.

The rest of the paper is organized as follows. Sect. 2 presents some lemmas and definitions for the proof. The proof of unique solvability is given in Sect. 3. And some conclusions are given in the final section.

Remark 1 Consider the unsteady 1D convection-diffusion equation

$$
\frac{\partial u}{\partial t}-p \frac{\partial^{2} u}{\partial x^{2}}+q(x, t) \frac{\partial u}{\partial x}=s(x, t), \quad(x, t) \in(0,1) \times(0, T]
$$

for the unknown transport variable $u(x, t)$. Here, $p>0$ is a constant diffusion coefficient, $q(x, t)$ is a variable convection coefficient, and $s(x, t)$ is a forcing function. Equation (2), which is often regarded as the linearized version of 1D Navier-Stokes equation, describes convection and diffusion of various physical properties such as mass, heat, energy, and vorticity. It is encountered in many fields of science and engineering [33, 34]. Therefore, it is of great importance to develop accurate and stable numerical methods for solving the convection-diffusion equations.

Concerning the time discretization of equation (2), the application of the backward Euler scheme leads to the semi-discrete scheme

$$
\frac{u^{n+1}-u^{n}}{\Delta t}-p \frac{\partial^{2} u^{n+1}}{\partial x^{2}}+q\left(x, t_{n+1}\right) \frac{\partial u^{n+1}}{\partial x}=s\left(x, t_{n+1}\right), \quad n=1,2, \ldots,
$$

where $\Delta t$ is time step size. For every time step $n$, equation (3) can be viewed as the differential equation like equation (1), with

$$
a(x)=\frac{q\left(x, t_{n+1}\right)}{p}, \quad b=\frac{1}{p \Delta t}>0, \quad c(x)=\frac{s\left(x, t_{n+1}\right)+u^{n} / \Delta t}{p} .
$$


Remark 2 For the unsteady multi-dimensional convection-diffusion problem, the alternating direction implicit (ADI) method can be adopted to convert it into a series of 1D problems [16-20].

\section{Preliminaries}

In this section, we will introduce some lemmas on circulant matrices which will be used to prove the main theorem in the next section.

Definition 1 ([35]) A circulant matrix $C$ is a Toeplitz matrix having the form

$$
C=\left[\begin{array}{ccccc}
c_{0} & c_{n-1} & \cdots & c_{2} & c_{1} \\
c_{1} & c_{0} & c_{n-1} & & c_{2} \\
\vdots & c_{1} & c_{0} & \ddots & \vdots \\
c_{n-2} & & \ddots & \ddots & c_{n-1} \\
c_{n-1} & c_{n-2} & \cdots & c_{1} & c_{0}
\end{array}\right]
$$

where each row is a cyclic shift of the row above it. The structure can also be characterized by noting that the $(k, j)$ entry of $C, C_{k, j}$, is given by

$$
C_{k, j}=c_{(j-k) \bmod n}
$$

Definition 2 ([23]) $\mathcal{C}_{n}^{3}(a, b, c)$ is an $n \times n$ circulant matrix determined by three elements $a, b, c$ and defined as follows:

$$
\mathcal{C}_{n}^{3}(a, b, c)=\left[\begin{array}{cccccc}
b & c & 0 & \cdots & 0 & a \\
a & b & c & 0 & \cdots & 0 \\
0 & a & b & c & \cdots & 0 \\
\vdots & \ddots & \ddots & \ddots & \ddots & \vdots \\
0 & \cdots & 0 & a & b & c \\
c & 0 & \cdots & 0 & a & b
\end{array}\right] \in \mathbb{R}^{n \times n}
$$

\section{Lemma 1}

$$
\mathcal{C}_{n}^{3}\left(a_{1}, a_{2}, a_{3}\right) \mathcal{C}_{n}^{3}\left(b_{1}, b_{2}, b_{3}\right)=\mathcal{C}_{n}^{5}\left(c_{1}, c_{2}, c_{3}, c_{4}, c_{5}\right),
$$

where

$$
\mathcal{C}_{n}^{5}\left(c_{1}, c_{2}, c_{3}, c_{4}, c_{5}\right)=\left[\begin{array}{cccccccc}
c_{3} & c_{4} & c_{5} & 0 & \cdots & 0 & c_{1} & c_{2} \\
c_{2} & c_{3} & c_{4} & \ddots & \ddots & \ddots & \ddots & c_{1} \\
c_{1} & c_{2} & c_{3} & \ddots & \ddots & \ddots & \ddots & 0 \\
0 & c_{1} & c_{2} & \ddots & \ddots & \ddots & \ddots & 0 \\
\vdots & \ddots & \ddots & \ddots & \ddots & \ddots & \ddots & \vdots \\
0 & \ddots & \ddots & \ddots & \ddots & \ddots & \ddots & c_{5} \\
c_{5} & \ddots & \ddots & \ddots & \ddots & c_{2} & c_{3} & c_{4} \\
c_{4} & c_{5} & 0 & \cdots & 0 & c_{1} & c_{2} & c_{3}
\end{array}\right] \in \mathbb{R}^{n \times n},
$$


and

$$
\begin{aligned}
& c_{1}=a_{1} b_{1}, \\
& c_{2}=a_{1} b_{2}+a_{2} b_{1}, \\
& c_{3}=a_{1} b_{3}+a_{2} b_{2}+a_{3} b_{1}, \\
& c_{4}=a_{2} b_{3}+a_{3} b_{2}, \\
& c_{5}=a_{3} b_{3} .
\end{aligned}
$$

Proof This lemma can be verified through direct computation.

Lemma 2 ([35]) For any two given circulant matrices $A$ and $B$, the sum $A+B$ is circulant, the product $A B$ is circulant, and two matrices $A, B$ commute, that is, $A B=B A$.

Lemma 3 ([36]) Assume that $A, B, C, D$ are $n \times n$ matrices. If $A C=C A$, then

$$
\left|\begin{array}{ll}
A & B \\
C & D
\end{array}\right|=|A D-C B| .
$$

If $B D=D B$, then

$$
\left|\begin{array}{ll}
A & B \\
C & D
\end{array}\right|=|D A-B C| .
$$

Remark 3 It is worth noting that if the matrix $D$ is invertible, this lemma can be easily verified through matrix computation. In 2000, Silvester obtained the above results even if $D$ is not invertible, which will be used in the proof of Theorem 1 .

\section{Unique solvability of CCD}

For a positive integer $M$, let $h=1 / M$. Discretize the interval $[0,1]$ into a uniform grid $0=x_{0}<x_{1}<\cdots<x_{M-1}<x_{M}=1$, where $x_{i}=i h, i=0, \ldots, M$. Denote the numerical approximations of $u\left(x_{i}\right), u_{x}\left(x_{i}\right), u_{x x}\left(x_{i}\right)$ by $U_{i}, U_{i}^{\prime}, U_{i}^{\prime \prime}$, respectively.

The CCD scheme for equation (1) with periodic boundary condition is given as follows [2]:

$$
\begin{aligned}
& \frac{7}{16}\left(U_{i+1}^{\prime}+U_{i-1}^{\prime}\right)+U_{i}^{\prime}-\frac{h}{16}\left(U_{i+1}^{\prime \prime}-U_{i-1}^{\prime \prime}\right)-\frac{15}{16 h}\left(U_{i+1}-U_{i-1}\right) \\
& \quad=0, \\
& \frac{9}{8 h}\left(U_{i+1}^{\prime}-U_{i-1}^{\prime}\right)+U_{i}^{\prime \prime}-\frac{1}{8}\left(U_{i+1}^{\prime \prime}+U_{i-1}^{\prime \prime}\right)-\frac{3}{h^{2}}\left(U_{i+1}-2 U_{i}+U_{i-1}\right) \\
& \quad=0,
\end{aligned}
$$

and

$$
-U_{i}^{\prime \prime}+a_{i} U_{i}^{\prime}+b U_{i}=c_{i}, \quad i=0,1, \ldots, M,
$$


where $a_{i}=a\left(x_{i}\right), c_{i}=c\left(x_{i}\right)$. And we use

$$
\begin{aligned}
& U_{-1}=U_{M-1}, \quad U_{-1}^{\prime}=U_{M-1}^{\prime}, \quad U_{-1}^{\prime \prime}=U_{M-1}^{\prime \prime}, \\
& U_{M+1}=U_{1}, \quad U_{M+1}^{\prime}=U_{1}^{\prime}, \quad U_{M+1}^{\prime \prime}=U_{1}^{\prime \prime} \text {. }
\end{aligned}
$$

In order to write the CCD system in a concise style, we order all the unknowns in the natural column-wise sense (unlike the way of gathering three unknowns at a grid point into a sub-block [3]),

$$
\mathbf{v}=\left[\begin{array}{c}
\mathbf{u}_{x} \\
\mathbf{u}_{x x} \\
\mathbf{u}
\end{array}\right]
$$

where the unknown vectors are

$$
\mathbf{u}=\left[U_{0}, U_{1}, \ldots, U_{M}\right]^{T}, \quad \mathbf{u}_{\mathbf{x}}=\left[U_{0}^{\prime}, U_{1}^{\prime}, \ldots, U_{M}^{\prime}\right]^{T}, \quad \mathbf{u}_{\mathbf{x x}}=\left[U_{0}^{\prime \prime}, U_{1}^{\prime \prime}, \ldots, U_{M}^{\prime \prime}\right]^{T}
$$

Let $n \triangleq M+1$, then we can rewrite the CCD system (7)-(8) as the following $3 \times 3$ block linear system:

$$
A \mathbf{u}=\left[\begin{array}{ccc}
A_{1,1} & A_{1,2} & A_{1,3} \\
A_{2,1} & A_{2,2} & A_{2,3} \\
\operatorname{diag}\left(a_{i}\right) & -E_{n} & b E_{n}
\end{array}\right] \cdot\left[\begin{array}{c}
\mathbf{u}_{x} \\
\mathbf{u}_{x x} \\
\mathbf{u}
\end{array}\right]=\left[\begin{array}{c}
\mathbf{0} \\
\mathbf{0} \\
\mathbf{c}
\end{array}\right]
$$

where

$$
\begin{array}{ll}
A_{1,1}=\mathcal{C}_{n}^{3}(7,16,7), & A_{1,2}=-h \mathcal{C}_{n}^{3}(-1,0,1), \quad A_{1,3}=-\frac{15}{h} \mathcal{C}_{n}^{3}(-1,0,1), \\
A_{2,1}=\frac{9}{h} \mathcal{C}_{n}^{3}(-1,0,1), & A_{2,2}=\mathcal{C}_{n}^{3}(-1,8,-1), \quad A_{2,3}=\frac{24}{h^{2}} \mathcal{C}_{n}^{3}(-1,2,-1),
\end{array}
$$

and $\mathbf{c}=\left\{c_{i}\right\}$ is a known vector, $\mathbf{0}$ is a zero vector of length $n$.

Theorem 1 Under the periodic boundary conditions, the CCD scheme (7)-(8) for equation (1) is uniquely solvable when

$$
h \leq \frac{1}{\frac{3}{4} \max \left\{\left|a_{i}\right|\right\}+\sqrt{\frac{5}{6} b+\frac{9}{16}\left(\max \left\{\left|a_{i}\right|\right\}\right)^{2}}} .
$$

Proof From Laplace's expansion theorem, we have

$$
\begin{aligned}
|A| & =\left|\begin{array}{ccc}
A_{1,1} & A_{1,2} & A_{1,3} \\
A_{2,1} & A_{2,2} & A_{2,3} \\
\operatorname{diag}\left(a_{i}\right) & -E_{n} & b E_{n}
\end{array}\right| \\
& =\left|\begin{array}{ccc}
A_{1,1}+A_{1,2} \operatorname{diag}\left(a_{i}\right) & A_{1,2} & A_{1,3}+b A_{1,2} \\
A_{2,1}+A_{2,2} \operatorname{diag}\left(a_{i}\right) & A_{2,2} & A_{2,3}+b A_{2,2} \\
\mathbf{0} & -E_{n} & \mathbf{0}
\end{array}\right|
\end{aligned}
$$




$$
\begin{aligned}
& =(-1)^{(2 n+1+2 n+2+\cdots+3 n)+(n+1+n+2+\cdots+2 n)}(-1)^{n}\left|\begin{array}{ll}
A_{1,1}+A_{1,2} \operatorname{diag}\left(a_{i}\right) & A_{1,3}+b A_{1,2} \\
A_{2,1}+A_{2,2} \operatorname{diag}\left(a_{i}\right) & A_{2,3}+b A_{2,2}
\end{array}\right| \\
& =\left|\begin{array}{ll}
A_{1,1}+A_{1,2} \operatorname{diag}\left(a_{i}\right) & A_{1,3}+b A_{1,2} \\
A_{2,1}+A_{2,2} \operatorname{diag}\left(a_{i}\right) & A_{2,3}+b A_{2,2}
\end{array}\right|
\end{aligned}
$$

Since $A_{1,3}, A_{1,2}, A_{2,3}$, and $A_{2,2}$ are circulant matrices, $A_{1,3}+b A_{1,2}$ and $A_{2,3}+b A_{2,2}$ are also circulant matrices, they can commute with each other from Lemma 2. Applying Lemma 1 and Lemma 3, we have

$$
\begin{aligned}
|A|= & \left|\left(A_{2,3}+b A_{2,2}\right)\left(A_{1,1}+A_{1,2} \operatorname{diag}\left(a_{i}\right)\right)-\left(A_{1,3}+b A_{1,2}\right)\left(A_{2,1}+A_{2,2} \operatorname{diag}\left(a_{i}\right)\right)\right| \\
= & \left|A_{1,1} A_{2,3}-A_{2,1} A_{1,3}+\left(A_{1,2} A_{2,3}-A_{2,2} A_{1,3}\right) \operatorname{diag}\left(a_{i}\right)+b\left(A_{1,1} A_{2,2}-A_{1,2} A_{2,1}\right)\right| \\
= & \mid \frac{1}{h^{2}} \mathcal{C}_{n}^{5}(-33,-48,162,-48,-33)+\frac{9}{h} \mathcal{C}_{n}^{5}(-1,-8,0,8,1) \operatorname{diag}\left(a_{i}\right) \\
& +2 b \mathcal{C}_{n}^{5}(1,20,48,20,1) \mid \\
\triangleq & |B|,
\end{aligned}
$$

where we have used that

$$
\begin{aligned}
A_{1,1} A_{2,3}-A_{2,1} A_{1,3} \\
=\mathcal{C}_{n}^{3}(7,16,7) \frac{24}{h^{2}} \mathcal{C}_{n}^{3}(-1,2,-1)+\frac{9}{h} \mathcal{C}_{n}^{3}(-1,0,1) \frac{15}{h} \mathcal{C}_{n}^{3}(-1,0,1) \\
=\frac{24}{h^{2}} \mathcal{C}_{n}^{5}(-7,-2,18,-2,-7)+\frac{135}{h^{2}} \mathcal{C}_{n}^{5}(1,0,-2,0,1) \\
=\frac{1}{h^{2}} \mathcal{C}_{n}^{5}(-33,-48,162,-48,-33), \\
A_{1,2} A_{2,3}-A_{2,2} A_{1,3} \\
=-h \mathcal{C}_{n}^{3}(-1,0,1) \frac{24}{h^{2}} \mathcal{C}_{n}^{3}(-1,2,-1)+\mathcal{C}_{n}^{3}(-1,8,-1) \frac{15}{h} \mathcal{C}_{n}^{3}(-1,0,1) \\
=-\frac{24}{h} \mathcal{C}_{n}^{5}(1,-2,0,2,-1)+\frac{15}{h} \mathcal{C}_{n}^{5}(1,-8,0,8,-1) \\
=\frac{9}{h} \mathcal{C}_{n}^{5}(-1,-8,0,8,1),
\end{aligned}
$$

and

$$
\begin{aligned}
A_{1,1} A_{2,2}-A_{1,2} A_{2,1} & =\mathcal{C}_{n}^{3}(7,16,7) \mathcal{C}_{n}^{3}(-1,8,-1)+h \mathcal{C}_{n}^{3}(-1,0,1) \frac{9}{h} \mathcal{C}_{n}^{3}(-1,0,1) \\
& =\mathcal{C}_{n}^{5}(-7,40,114,40,-7)+9 \mathcal{C}_{n}^{5}(1,0,-2,0,1) \\
& =2 \mathcal{C}_{n}^{5}(1,20,48,20,1) .
\end{aligned}
$$

Obviously, the matrix $B$ has at most five nonzero elements per column. The main diagonal element

$$
B_{j, j}=\frac{162}{h^{2}}+96 b,
$$


and the other four nonzero elements in $j$-column are

$$
\begin{aligned}
& B_{(j-1) \bmod n, j}=-\frac{48}{h^{2}}+40 b+\frac{72}{h} a_{j}, \\
& B_{(j+1) \bmod n, j}=-\frac{48}{h^{2}}+40 b-\frac{72}{h} a_{j}, \\
& B_{(j-2) \bmod n, j}=-\frac{33}{h^{2}}+2 b+\frac{9}{h} a_{j}, \\
& B_{(j+2) \bmod n, j}=-\frac{33}{h^{2}}+2 b-\frac{9}{h} a_{j} .
\end{aligned}
$$

If

$$
\left\{\begin{array}{l}
-\frac{48}{h^{2}}+40 b+\frac{72}{h} \max \left\{\left|a_{j}\right|\right\} \leq 0 \\
-\frac{33}{h^{2}}+2 b+\frac{9}{h} \max \left\{\left|a_{j}\right|\right\} \leq 0
\end{array}\right.
$$

then

$$
\begin{aligned}
\sum_{i=1,2, \ldots, n, i \neq j}\left|B_{i, j}\right|= & \left|B_{(j-1) \bmod n, j}\right|+\left|B_{(j+1) \bmod n, j}\right|+\left|B_{(j-2) \bmod n, j}\right|+\left|B_{(j+2) \bmod n, j}\right| \\
= & \left(\frac{48}{h^{2}}-40 b-\frac{72}{h} a_{j}\right)+\left(\frac{48}{h^{2}}-40 b+\frac{72}{h} a_{j}\right) \\
& +\left(\frac{33}{h^{2}}-2 b-\frac{9}{h} a_{j}\right)+\left(\frac{33}{h^{2}}-2 b+\frac{9}{h} a_{j}\right) \\
= & \frac{162}{h^{2}}-84 b \\
< & \frac{162}{h^{2}}+96 b=B_{j, j}
\end{aligned}
$$

where we have used that $b>0$. Thus the matrix $B$ is strictly diagonally dominant by columns, and $|A|=|B| \neq 0$.

Indeed, inequalities (12) are equivalent to

$$
\frac{6}{h^{2}}-\frac{9 \max \left\{\left|a_{j}\right|\right\}}{h}-5 b=6\left(\frac{1}{h}-\frac{3}{4} \max \left\{\left|a_{i}\right|\right\}\right)^{2}-5 b-\frac{27}{8}\left(\max \left\{\left|a_{i}\right|\right\}\right)^{2} \geq 0 .
$$

It is easy to check that the above inequality holds for

$$
h \leq \frac{1}{\frac{3}{4} \max \left\{\left|a_{i}\right|\right\}+\sqrt{\frac{5}{6} b+\frac{9}{16}\left(\max \left\{\left|a_{i}\right|\right\}\right)^{2}}} .
$$

Therefore, when $h$ satisfies condition (14), the CCD scheme for equation (1) is uniquely solvable. This completes the proof of the theorem.

\section{Conclusions}

A theoretical proof for the unique solvability of the CCD system for solving the 1D convection-diffusion equation with variable convection coefficients subject to the periodic boundary conditions is given in this paper. 
The CCD method can be directly used to solve the following 2D/3D unsteady convectiondiffusion equations without mixed derivative by combining with the ADI method [16, 23],

$$
\frac{\partial u}{\partial t}-p \frac{\partial^{2} u}{\partial x^{2}}-q \frac{\partial^{2} u}{\partial y^{2}}+a(x, y, t) \frac{\partial u}{\partial x}+b(x, y, t) \frac{\partial u}{\partial y}=s(x, y, t)
$$

where $p, q$ are positive diffusion coefficients, and $a(x, y), b(x, y)$ are variable convection coefficients in $x$ - and $y$-directions, respectively. Therefore, the unique solvability of numerical solution of the CCD method for solving the above 2D/3D unsteady convectiondiffusion equations subject to periodic boundary conditions can also be obtained. However, the results obtained in this paper cannot be directly adopted for the fractional order case as given in $[37,38]$.

This paper only focuses on the unique solvability of the CCD system for solving the convection-diffusion equation. Our future works will be focused on the convergence analysis of the CCD method and the generalization of the method for fractional order cases. And the CCD method for solving multi-dimensional elliptic boundary value problems [39-42] is also our future objective.

\section{Acknowledgements}

Kejia Pan was supported by the National Natural Science Foundation of China (No. 41474103), the Excellent Youth Foundation of Hunan Province of China (No. 2018JJ1042), the Natural Science Foundation of Hunan Province of China (No. 2015JJ3148) and the Innovation-Driven Project of Central South University (No. 2018CX042). Hongling Hu was supported by the program for excellent talents in Hunan Normal University (No. ET1501). We are grateful to the three anonymous reviewers for their helpful comments.

\section{Competing interests}

The authors declare that they have no competing interests.

\section{Authors' contributions}

All authors contributed equally and significantly in writing this article. All authors read and approved the final manuscript.

\section{Author details}

${ }^{1}$ School of Mathematics and Statistics, Central South University, Changsha, P.R. China. ${ }^{2}$ School of Mathematics and Statistics, Key Laboratory of High Performance Computing and Stochastic Information Processing, Hunan Normal University, Changsha, P.R. China.

\section{Publisher's Note}

Springer Nature remains neutral with regard to jurisdictional claims in published maps and institutional affiliations.

Received: 27 December 2017 Accepted: 5 April 2018 Published online: 04 May 2018

\section{References}

1. Lele, S.K.: Compact finite difference schemes with spectral-like resolution. J. Comput. Phys. 103, 16-42 (1992)

2. Chu, P.C., Fan, C.: A three-point combined compact difference scheme. J. Comput. Phys. 140, 370-399 (1998)

3. Chu, P.C., Fan, C.: A three-point sixth-order nonuniform combined compact difference scheme. J. Comput. Phys. 148, 663-674 (1999)

4. Chu, P.C., Fan, C.: A three-point sixth-order staggered combined compact difference scheme. Math. Comput. Model. $32,323-340(2000)$

5. Nihei, T., Ishii, K.: A fast solver of the shallow water equations on a sphere using a combined compact difference scheme. J. Comput. Phys. 187, 639-659 (2003)

6. Chen, W., Chen, J.C., Lo, E.Y.: An interpolation based finite difference method on non-uniform grid for solving Navier-Stokes equations. Comput. Fluids 101, 273-290 (2014)

7. Ghader, S., Nordström, J.: High-order compact finite difference schemes for the vorticity-divergence representation of the spherical shallow water equations. Int. J. Numer. Methods Fluids 78, 709-738 (2015)

8. Cui, M.: Combined compact difference scheme for the time fractional convection-diffusion equation with variable coefficients. Appl. Math. Comput. 246, 464-473 (2014)

9. Takahashi, F.: Implementation of a high-order combined compact difference scheme in problems of thermally driven convection and dynamo in rotating spherical shells. Geophys. Astrophys. Fluid Dyn. 106, 231-249 (2012)

10. Gao, G.H., Sun, H.W.: Three-point combined compact difference schemes for time-fractional advection-diffusion equations with smooth solutions. J. Comput. Phys. 298, 520-538 (2015) 
11. Sengupta, T.K., Lakshmanan, V., Vijay, V:: A new combined stable and dispersion relation preserving compact scheme for non-periodic problems. J. Comput. Phys. 228, 3048-3071 (2009)

12. Yu, C.H., Wang, D., He, Z.T.: Pähtz. An optimized dispersion-relation-preserving combined compact difference scheme to solve advection equations. J. Comput. Phys. 300, 92-115 (2015)

13. Yu, C.H., Bhumkar, Y.G., Sheu, T.W.H.: Dispersion relation preserving combined compact difference schemes for flow problems. J. Sci. Comput. 62, 482-516 (2015)

14. Yu, C.H., Sheu, T.W.H.: Development of a combined compact difference scheme to simulate soliton collision in a shallow water equation. Commun. Comput. Phys. 19, 603-631 (2016)

15. Sengupta, T.K., Vijay, V., Bhaumik, S.: Further improvement and analysis of CCD scheme: dissipation discretization and de-aliasing properties. J. Comput. Phys. 228, 6150-6168 (2009)

16. Sun, H.W., Li, L.Z.: A CCD-ADI method for unsteady convection-diffusion equations. Comput. Phys. Commun. 185, 790-797 (2014)

17. Li, L.Z., Sun, H.W., Tam, S.C.: A spatial sixth-order alternating direction implicit method for two-dimensional cubic nonlinear Schrödinger equations. Comput. Phys. Commun. 187, 38-48 (2015)

18. Gao, G.H., Sun, H.W.: Three-point combined compact alternating direction implicit difference schemes for two-dimensional time-fractional advection-diffusion equations. Commun. Comput. Phys. 17, 487-509 (2015)

19. He, D.D.: An unconditionally stable spatial sixth-order CCD-ADI method for the two-dimensional linear telegraph equation. Numer. Algorithms 72, 1103-1117 (2016)

20. He, D.D., Pan, K.J.: An unconditionally stable linearized CCD-ADI method for generalized nonlinear Schrodinger equations with variable coefficients in two and three dimensions. Comput. Math. Appl. 73, 2360-2374 (2017)

21. He, D.D., Pan, K.J.: A fifth-order combined compact difference scheme for the Stokes flow on polar geometries. East Asian J. Appl. Math. 7, 714-727 (2018)

22. Chen, B.Y., He, D.D., Pan, K.J.: A linearized high-order combined compact difference scheme for multi-dimensional coupled Burgers' equations. Numer. Math., Theory Methods Appl. 11, 299-320 (2018)

23. Lee, S.T., Liu, J., Sun, H.W.: Combined compact difference scheme for linear second-order partial differential equations with mixed derivative. J. Comput. Appl. Math. 264, 23-37 (2014)

24. Kumar, D., Singh, J., Baleanu, D.: A new numerical algorithm for fractional Fitzhugh-Nagumo equation arising in transmission of nerve impulses. Nonlinear Dyn. 91, 307-317 (2018)

25. Kumar, D., Agarwal, R.P., Singh, J: A modified numerical scheme and convergence analysis for fractional model of Lienard's equation. J. Comput. Appl. Math. 339, 405-413 (2018)

26. Singh, H., Srivastava, H.M., Kumar, D.: A reliable numerical algorithm for the fractional vibration equation. Chaos Solitons Fractals 103, 131-138 (2017)

27. Singh, J., Kumar, D., Qurashi, M.A., et al.: A novel numerical approach for a nonlinear fractional dynamical model of interpersonal and romantic relationships. Entropy 19, 375 (2017)

28. Yang, X.J., Gao, F., Srivastava, H.M.: A new computational approach for solving nonlinear local fractional PDEs. J. Comput. Appl. Math. 339, 285-296 (2018)

29. Yang, X.J., Gao, F., Srivastava, H.M.: Exact travelling wave solutions for the local fractional two-dimensional Burgers-type equations. Comput. Math. Appl. 73, 203-210 (2017)

30. Yang, X.J., Cao, F.: A new technology for solving diffusion and heat equations. Therm. Sci. 21, 133-140 (2017)

31. He, D.D., Pan, K.J.: An unconditionally stable linearized difference scheme for the fractional Ginzburg-Landau equation. Numer. Algorithms (2018). https://doi.org/10.1007/s11075-017-0466-y

32. Zhang, J., Zhao, J.J.: Truncation error and oscillation property of the combined compact difference scheme. Appl. Math. Comput. 161, 241-251 (2005)

33. Hirsh, R.S.: Higher order accurate difference solutions of fluid mechanics problems by a compact differencing technique. J. Comput. Phys. 19, 90-109 (1975)

34. Tian, Z.F.: A rational high-order compact ADI method for unsteady convection-diffusion equations. Comput. Phys. Commun. 182, 649-662 (2011)

35. Davis, P.J.: Circulant Matrices. Am. Math. Soc., Providence (2012)

36. Silvester, J.R.: Determinants of block matrices. Math. Gaz. 84, 460-467 (2000)

37. Yang, X.J., Baleanu, D., Khan, Y., et al.: Local fractional variational iteration method for diffusion and wave equations on Cantor sets. Rom. J. Phys. 59, 36-48 (2014)

38. Bhrawy, A.H., Baleanu, D.: A spectral Legendre-Gauss-Lobatto collocation method for a space-fractional advection diffusion equations with variable coefficients. Rep. Math. Phys. 72, 219-233 (2013)

39. Pan, K.J., He, D.D., Hu, H.L., Ren, Z.Y.: A new extrapolation cascadic multigrid method for three dimensional elliptic boundary value problems. J. Comput. Phys. 344, 499-515 (2017)

40. Pan, K.J., He, D.D., Hu, H.L.: An extrapolation cascadic multigrid method combined with a fourth-order compact scheme for 3D Poisson equation. J. Sci. Comput. 70, 1180-1203 (2017)

41. Pan, K.J., He, D.D., Chen, C.M.: An extrapolation cascadic multigrid method for elliptic problems on reentrant domains. Adv. Appl. Math. Mech. 9, 1347-1363 (2017)

42. Hu, H.L., Ren, Z.Y., et al.: On the convergence of an extrapolation cascadic multigrid method for elliptic problems. Comput. Math. Appl. 74, 759-771 (2017) 